\title{
Is complement the culprit behind COVID-19 vaccine- related adverse reactions?
}

\author{
Dimitrios C. Mastellos, ${ }^{1}$ Panagiotis Skendros, ${ }^{2}$ and John D. Lambris ${ }^{3}$ \\ 'National Center for Scientific Research 'Demokritos', Athens, Greece. 'First Department of Internal Medicine and Laboratory of Molecular Hematology, University Hospital of Alexandroupolis, Democritus \\ University of Thrace, Alexandroupolis, Greece. ³epartment of Pathology and Laboratory Medicine, Perelman School of Medicine, University of Pennsylvania, Philadelphia, Pennsylvania, USA.
}

\section{Rare thrombotic reactions to adenovirus-based COVID-19 vaccines}

Undeniably, a global and coordinated COVID-19 vaccination effort is a prerequisite for taming the spread of emerging SARS-CoV-2 variants and achieving population-wide immunity that can thwart future viral surges. Ongoing vaccination programs have relied on both mRNA and adenoviral-based formulations eliciting potent humoral responses and showing safety in the majority of the population vaccinated so far. However, rare vaccine-related adverse events were recently reported in individuals who received the adenoviral-encoded ChAdOx1 nCov-19 and Ad26.COV2.S vaccines (1-3). Most of these cases developed acute thrombotic complications (cerebral venous thrombosis or disseminated intravascular coagulation) and severe thrombocytopenia following the first dose of the vaccine, without any prior history of heparin exposure or known risk factors for thrombophilia. This rare syndrome, termed vaccine-induced immune thrombotic thrombocytopenia (VITT), clinically resembles the hallmarks of autoimmune heparin-induced thrombocytopenia (autoimmune HIT) and is linked to the consistent presence of circulating autoantibodies against platelet factor 4 (PF4), which can directly activate platelets in the absence of heparin (2).

The prothrombotic action of anti-PF4 antibodies likely involves antibody-medi- ated platelet activation through IgG-Fc $\gamma \mathrm{R}$ interactions and similar FcR-mediated engagement of immune effector cells such as monocytes/macrophages and neutrophils (Figure 1). Since VITT is a newly described syndrome, its clinical management mostly relies on its similarities with HIT and non-heparin-dependent autoimmune thrombotic thrombocytopenias. While nonheparin anticoagulants and intravenous immunoglobulin (IVIg) have been proposed as first-line treatments for patients with VITT, these approaches are not fail-safe and entail drawbacks that may curtail their effectiveness (e.g., risk of promiscuous FcR activation, acute anaphylaxis, or even thromboembolic events in the case of intravenous immunoglobulin) (4). Several second-line therapies for HIT have also been suggested, including plasmapheresis, especially when nonheparin anticoagulation is contraindicated because of major bleeding events (5). Thus, more insight into the precise pathophysiology of VITT is needed to enable more personalized medicine approaches.

\section{A proposed role for complement}

Complement is a host innate immune sentinel comprising over 50 proteins that can be swiftly activated through any of three initiating pathways (classical, lectin, or alternative) that sense microbial invaders or other inflammatory cues, such as immune complexes. In fact, comple-
Conflict of interest: JDL is the founder of Amyndas Pharmaceuticals, which is developing complement inhibitors for therapeutic purposes. He is the inventor on patents or patent applications that describe the use of complement inhibitors for therapeutic purposes, some of which are developed by Amyndas Pharmaceuticals: US patents 8946145/9371365 (Modified compstatin with peptide backbone and C-terminal modifications), 9630992 (Compstatin analogs with improved pharmacokinetic properties), 9579360 (Methods of treating or preventing periodontitis and diseases associated with periodontitis). JDL is also the inventor of the compstatin technology licensed to Apellis Pharmaceuticals, i.e., 4(1MeW)7W/POT-4/APL-1 and PEGylated derivatives such as APL-2/pegcetacoplan/ empaveli and APL-9: US patents 6319897 (Peptides which inhibit complement activation), 7989589 (Compstatin analogs with improved activity), 7888323 (Potent compstatin analogs).

Copyright: () 2021, American Society for Clinical Investigation.

Reference information: / Clin Invest. 2021;131(11):e151092. https://doi.org/10.1172/JCI151092. ment activation has been implicated as a perpetrator of immune complex-driven thromboinflammation in autoimmune pathologies such as the antiphospholipid syndrome, a systemic disorder that increases risk for blood clots (6). Prompted by the striking findings in patients who developed VITT after a single dose of adenoviral-associated vaccine, we posit here that the observed thrombotic complications may partly reflect immune complextriggered complement activation through the classical pathway. PF4-containing immune complexes can be recognized by $\mathrm{C} 1 \mathrm{q}$, a soluble pattern recognition molecule of the classical pathway that binds to the Fc portion of IgG molecules. Anti-PF4 complex-driven classical pathway activation can lead to C3 activation, alternative pathway-mediated amplification of complement responses, and downstream generation of potent proinflammatory mediators and effectors (C3a, C5a and membrane attack complex) that can potentiate thromboinflammation (7). In fact, the thrombogenic capacity of distinct complement activation fragments has been linked to a broad spectrum of immune-related mechanisms that underpin the immunothrombosis of severe COVID-19 $(7,8)$.

Considering that certain allelic combinations of complement gene polymorphisms within the population confer differential susceptibility to deregulated complement activity, it is tempting to speculate that these vaccinated individuals may represent cases where complement deregulation occurs due to genetic alterations that result in ineffective host regulatory control (9). Deregulated complement responses can perpetuate a vicious cycle of inflammatory tissue damage that renders the vascular endothelium and platelet/ monocyte compartment more thrombogenic. In this regard, circulating anti-PF4 immune complexes may well serve as a first hit that ignites complement acti- 


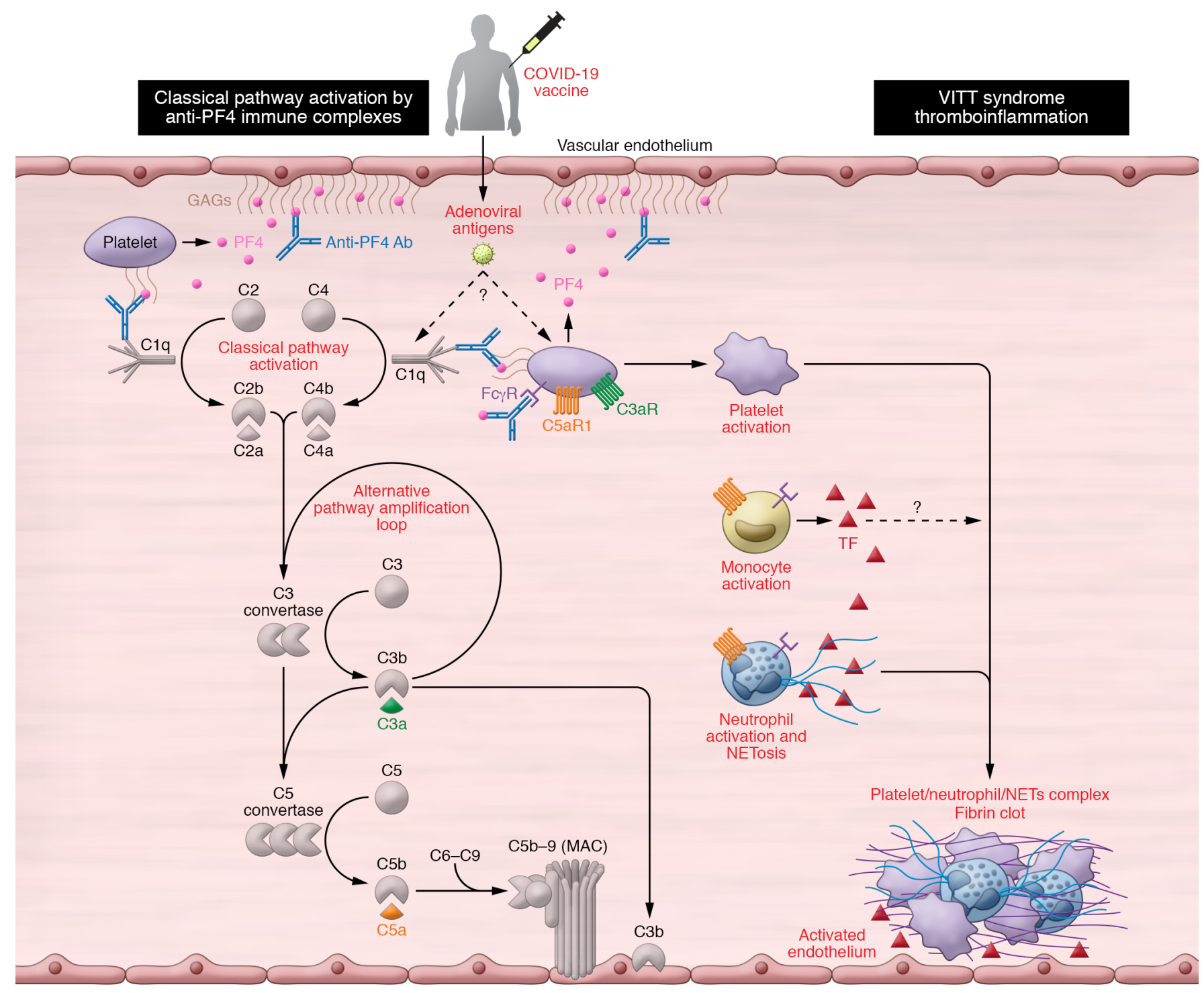

Figure 1. A schematic diagram of the plausible mechanisms by which complement may contribute to the VITT-associated prothrombotic response. Vaccine-induced immune thrombotic thrombocytopenia (VITT) has been described in individuals presenting with high titers of platelet-activating anti-platelet factor 4 (PF4) autoantibodies. These antibodies recognize large multiantigenic complexes comprising PF4 and polyanionic structures similar to heparin, and resemble autoimmune heparin-induced thrombocytopenia (HIT). Complement activation can be triggered in multiple ways in patients presenting with VITT and anti-PF4 antibodies. First, the formation of complexes between PF4 and certain vaccine constituents (e.g., adenoviral capsid proteins or DNA) (23) may serve as a scaffold for C3 activation, similarly to what has been observed with PF4-heparin ultra-large complexes. In addition, complement activation can be triggered by the binding of C1q to the anti-PF4 immune complexes that are deposited on the endothelium, monocyte, or platelet surface via binding to polyanionic structures such as glycosaminoglycans (GAGs). Classical pathway activation leads to C3 cleavage, amplification of complement responses via the alternative pathway, downstream generation of proinflammatory C3a and C5a anaphylatoxins, and the formation of the C5b-9 complex. Complement activation fragments mediate a broad range of thromboinflammatory reactions by interacting with complement receptors on platelets, monocytes, and neutrophils. These interactions can induce or enhance FcR-mediated platelet activation, neutrophil-platelet aggregation, and release of TF-loaded neutrophil extracellular traps (NETs) from activated neutrophils. All these processes are fueled by complement activation and can collectively contribute to a prothrombotic environment that may lead to VITT. Moreover, C3-opsonized immune complexes can enhance Fc $\gamma \mathrm{R}$-dependent effector responses on platelets further promoting thrombotic responses. Abbreviations: CP, classical pathway; AP, alternative pathway; TF, tissue factor.

vation, in a way similar to complementmediated thrombotic microangiopathies (10). The fact that a fraction of these antiPF4 autoantibodies may recognize antigenic complexes between PF4 and polyanionic structures (such as GAGs or heparan sulfate) raises the possibility that these immune complexes may skew the binding of endogenous complement regulators to host polyanionic surfaces, thereby altering the capacity of these surfaces to withstand autologous complement attack.
C3 activation may play a pivotal pathogenic role in this vaccine-induced immunothrombotic phenotype. C3-opsonized anti-PF4 immune complexes can enhance the phagocytic and inflammatory properties of monocytes and neutrophils through 
the synergistic engagement of $\mathrm{F} c \gamma \mathrm{R}$ and complement receptors, thereby increasing the thrombogenic milieu in the vasculature (Figure 1). C3 fragment deposition on the endothelium and on platelets/neutrophils can potentiate thromboinflammatory responses by promoting CR3-dependent platelet-neutrophil interactions that favor platelet adhesion and clotting (11). Also, C3aR signaling on platelets can further drive thrombotic responses (12). Of note, it has been shown that PF4-heparin complexes, a hallmark of HIT pathology, can potently activate $\mathrm{C} 3$ in the fluid phase and C3 inhibitors such as Cp40 can abrogate C3 activation triggered by HIT-associated PF4-heparin complexes (13). In line with this, the Cp40-based drug candidate AMY-101 has been shown to effectively decrease both neutrophils and neutrophil extracellular trap (NET) formation, two major drivers of HIT-associated thrombosis, in patients with COVID-19 (7, 14-16).

\section{Potential treatment approaches}

In an analogy to HIT, clinical-stage complement C3 inhibitors may represent a novel therapeutic strategy for ameliorating the thrombotic complications elicited in VITT. On one hand, C3 inhibition may quench anti-PF4 antibody production by reducing antigen uptake or processing in the lymphoid tissue. On the other hand, it can broadly suppress multiple paths of immune complex-driven thromboinflammation in VITT (Figure 1). Moreover, C3 inhibition may offer broader therapeutic control of thromboembolic reactions mediated by pathogenic autoantibodies. In this regard, C3 activation, but not C5, was shown to be indispensable for antiphospholipid antibody-induced tissue factor (TF) activation and thrombosis in antiphospholipid syndrome (17). The recently reported resolution of vaccine-induced thrombotic microangiopathy in two patients following combined treatment with anticoagulants and the C5 inhibitor eculizumab further corroborates our hypothesis that therapeutic complement modulation may offer significant clinical benefits to patients with VITT (18). In this regard, C3 inhibitors may exert a broader therapeutic effect than anti-C5 agents by suppressing both terminal pathway activation and thrombogenic pathways operating upstream of C5 $(14,19)$.
Because this newly described immune syndrome is still poorly defined, it would be of great benefit to patients with VITT if the algorithm for VITT clinical management considered diagnostic monitoring of complement biomarkers as a means of validating the use of specific complement inhibitors. As a cautionary note, we should stress that plasma levels of complement proteins reflect both turnover and synthesis. Therefore, the accurate monitoring of complement activity should rely on markers of ongoing activation combined with corroborative functional assays that gauge the impact of complement activation on the tissue or cellular level.

\section{mRNA vaccine reactions}

In addition to these cases of VITT in individuals receiving adenovirus vectored vaccines, the rollout of COVID-19 mRNA vaccines has brought to the spotlight rare adverse events involving a maladaptive immune reaction to certain vaccine constituents. Rare cases of severe allergic reactions (anaphylaxis) were reported following the first dose of the Pfizer-BioNTech or Moderna SARS-CoV-2 mRNA vaccines (20). Such allergic reactions are likely triggered by the PEG or lipid moieties of these mRNA vaccines. They involve complement and mast cell activation in an IgE-independent manner and closely resemble the complement mediated pseudo-allergy reaction (CARPA) that has been described in the case of liposomal carriers (21). Complement activation in this case may be initiated by IgG or IgM directed against the PEGylated lipids of these vaccine formulations.

\section{Concluding remarks}

Taken together, these paradigms of vaccine-related adverse reactions highlight the potential contribution of complement activation to vaccine-related pathology, raise the importance of incorporating complement diagnostic monitoring in the early clinical management of such patients, and endorse complement inhibitors as potential treatment options. In addition to the complement-activating capacity of antiPF4 antibodies, we cannot rule out the possibility that complement activation in VITT may also be fueled by preformed (natural IgM) or induced IgG antibodies against adenoviral capsid antigens or direct inter- action of pattern recognition molecules with capsid elements (22). The relevance of these plausible mechanisms to VITT pathology warrants further investigation.

Address correspondence to: John D. Lambris, Department of Pathology \& Laboratory Medicine, 401 Stellar-Chance Laboratories, 422 Curie Boulevard, Philadelphia, Pennsylvania 19104, USA. Phone: 215. 746.5765; Email: lambris@pennmedicine. upenn.edu.

1. Muir K-L, et al. Thrombotic thrombocytopenia after Ad26.COV2.S vaccination [published online April 14, 2021]. N Engl J Med. https://doi. org/10.1056/NEJMc2105869.

2. Scully M, et al. Pathologic antibodies to platelet factor 4 after ChAdOx1 nCoV-19 vaccination [published online April 16, 2021]. N Engl J Med. https://doi.org/10.1056/NEJMoa2105385.

3. Schultz NH, et al. Thrombosis and thrombocytopenia after ChAdOx1 nCoV-19 vaccination [published online April 9, 2021]. N Engl J Med. https:// doi.org/10.1056/NEJMoa2104882.

4. Kapoor M, et al. Thromboembolic risk with IVIg: incidence and risk factors in patients with inflammatory neuropathy. Neurology. 2020;94(6):e635-e638.

5. Marchetti M, et al. Heparin-induced thrombocytopenia: a review of new concepts in pathogenesis, diagnosis, and management. JClin Med. 2021;10(4):683.

6. Chaturvedi S, et al. Complement in the pathophysiology of the antiphospholipid syndrome. Front Immunol. 2019;10:449.

7. Skendros $\mathrm{P}$, et al. Complement and tissue factorenriched neutrophil extracellular traps are key drivers in COVID-19 immunothrombosis. J Clin Invest. 2020;130(11):6151-6157.

8. Risitano AM, et al. Complement as a target in COVID-19? Nat Rev Immunol. 2020;20(6):343-344.

9. Heurich M, et al. Common polymorphisms in C3, factor $\mathrm{B}$, and factor $\mathrm{H}$ collaborate to determine systemic complement activity and disease risk. Proc Natl Acad Sci U S A. 2011;108(21):8761-8766.

10. Gavriilaki E, Brodsky RA. Complementopathies and precision medicine. JClin Invest. 2020;130(5):2152-2163.

11. Hamad OA, et al. Contact activation of $\mathrm{C} 3$ enables tethering between activated platelets and polymorphonuclear leukocytes via CD11b/ CD18. Thromb Haemost. 2015;114(6):1207-1217.

12. Sauter RJ, et al. Functional relevance of the anaphylatoxin receptor $\mathrm{C} 3 \mathrm{aR}$ for platelet function and arterial thrombus formation marks an intersection point between innate immunity and thrombosis. Circulation. 2018;138(16):1720-1735.

13. Khandelwal S, et al. Novel immunoassay for complement activation by PF4/heparin complexes. Thromb Haemost. 2018;118(8):1484-1487.

14. Mastellos DC, et al. Complement C3 vs C5 inhibition in severe COVID-19: early clinical findings reveal differential biological efficacy. Clin Immunol. 2020;220:108598. 
15. Gollomp K, et al. Neutrophil accumulation and NET release contribute to thrombosis in HIT. JCI Insight. 2018;3(18):99445.

16. Perdomo J, et al. Neutrophil activation and NETosis are the major drivers of thrombosis in heparin-induced thrombocytopenia. Nat Commun. 2019;10(1):1322.

17. Müller-Calleja N, et al. Complement C5 but not $\mathrm{C} 3$ is expendable for tissue factor activation by cofactor-independent antiphospholipid antibodies. Blood Adv. 2018;2(9):979-986.

18. Tiede A, et al. Prothrombotic immune thrombo- cytopenia after COVID-19 vaccine [published online April 30, 2021]. Blood. https://doi. org/10.1182/blood.2021011958.

19. Hillmen P, et al. Pegcetacoplan versus eculizum$\mathrm{ab}$ in paroxysmal nocturnal hemoglobinuria. N Engl J Med. 2021;384(11):1028-1037.

20. Castells MC, Phillips EJ. Maintaining safety with SARS-CoV-2 vaccines. $N$ Engl J Med. 2021;384(7):643-649.

21. Klimek L, et al. Allergenic components of the mRNA-1273 vaccine for COVID-19: possible involvement of polyethylene glycol and IgG- mediated complement activation [published online March 3, 2021]. Allergy. https://doi. org/10.1111/all.14794.

22. Chéneau C, Kremer EJ. Adenovirus-extracellular protein interactions and their impact on innate immune responses by human mononuclear phagocytes. Viruses. 2020;12(12):1351.

23. Greinacher A, et al. Towards understanding ChAdOx1 nCov-19 vaccine-induced immune thrombotic thrombocytopenia (VITT) [preprint]. https://doi.org/10.21203/rs.3.rs-440461/ v1. Posted on Research Square April 20, 2021. 